\title{
PERSPECTIVE OPEN Straintronics with van der Waals materials
}

\author{
Feng Miao $\mathbb{D}^{1 凶}$, Shi-Jun Liang ${ }^{1}$ and Bin Cheng $\mathbb{D}^{1,2 凶}$
}

With the outstanding mechanical properties, van der Waals (vdW) materials have attracted extensive attention in the research of straintronics in the past decade. In this perspective, we first review the recent progresses of the straintronics with vdW materials based on three different lattice deformation modes, i.e., in-plane strain, out-of-plane strain, and heterostrain. Then we discuss the current technique challenges in this field, and finally provide our perspectives on future research directions for both fundamental physics and electronic applications.

npj Quantum Materials (2021)6:59; https://doi.org/10.1038/s41535-021-00360-3

Tuning electronic properties of materials lies at the heart of modern electronics. Strain engineering, as an efficient strategy to tune the electronic properties of materials and explore new quantum states in condensed matter physics, gives rise to an emerging research field referred as "straintronics". In the past few years, straintronics with van der Waals (vdW) materials has attracted lots of attention ${ }^{1-11}$. VdW materials usually possess extraordinary mechanical properties, such as large anisotropic compressibility and Young's modulus, providing a powerful knob for both exploring novel quantum states and developing new device functionalities based on strain engineering. In light of the layered structure, the strains acting on vdW materials are usually classified into in-plane type and out-of-plane type (Fig. 1), which are achieved by stretching the sample along the two-dimensional (2D) plane and compressing the sample perpendicularly to the 2D plane, respectively. Furthermore, stacking vdW materials into vertical heterostructures leads to a new type of strain engineering, i.e., heterostrain. Here, we briefly review the recent progresses of straintronics with vdW materials according to different modes of lattice deformation, and share our perspectives on future research directions in this field.

As the mostly studied type of strain on vdW materials, in-plane strain has been widely utilized to engineer the electronic band structure ${ }^{12-16}$ through changing the covalent bond lengths and angles within individual 2D layer. Especially, the band gap modification controlled by in-plane strain has been first proposed in graphene ${ }^{17}$, and then achieved in transition metal dichalcogenides $(T M D s)^{18}$, black phosphorous ${ }^{19}$, etc. Besides, the deformation of in-plane lattice can also modulate the intralayer exchange energy $^{20}$ and spin-orbit coupling $(\mathrm{SOC})^{21}$, enabling the strainassisted transition between different magnetic states in the vdW magnets ${ }^{22}$. One recent example is ultra-sensitive magnetization reversal assisted by in-plane strain in $\mathrm{Fe}_{2} \mathrm{GeTe}_{3}{ }^{23}$, which indicates an alternative way to realize low-power magnetization switching in spintronic devices. In addition, transition from ferromagnetic state to antiferromagnetic state under in-plane strain in monolayer $\mathrm{Crl}_{3}$ is also predicted ${ }^{24,25}$, which requires more experimental efforts in the future. Another degree of freedom that could be adjusted by in-plane strain is the symmetry of crystal lattices. Especially, the rotational symmetry of vdW materials could be lowered if the in-plane strain is anisotropic, providing a unique approach to create the Berry curvature dipole and current induced magnetization in $\mathrm{vdW}$ materials with three-fold rotational symmetry, such as $\mathrm{MoS}_{2}{ }^{26}$, $\mathrm{BiTel}^{27}$, etc. Moreover, in-plane strain could alter the topology of the quantum states that is correlated to the rotational symmetry, enabling the topological switching in vdW topological materials. At the current stage, vdW topological materials that could be investigated by applying in-plane strain include anomalous quantum Hall insulators such as $\mathrm{MnBi}_{2} \mathrm{Te}_{4}{ }^{28}$ and magnetic twisted bilayer graphene 29,30 , quantum spin Hall insulator such as undoped monolayer $\mathrm{WTe}_{2}{ }^{31}$, and $2 \mathrm{D}$ topological crystalline insulators such as monolayer IV-VI semiconductors ${ }^{32}$. Finally, in-plain strain could significantly modify the electronic correlation effects, resulting in metal-to-insulator transition, modulating the charge-density-wave, and tuning superconducting properties, etc. In particular, uniaxial in-plane strain has been proved to be an effective tool to explore the superconducting pairing symmetry ${ }^{33}$, which could be utilized in vdW superconductors such as twisted bilayer graphene ${ }^{34}$, twisted double bilayer graphene ${ }^{35}$, monolayer $\mathrm{NbSe}_{2}{ }^{36}$, doped monolayer $\mathrm{WTe}_{2}{ }^{37}$, monolayer $\mathrm{Fe}(\mathrm{Te}, \mathrm{Se})^{38}$, and monolayer $\mathrm{Bi}_{2} \mathrm{Sr}_{2} \mathrm{CaCu}_{2} \mathrm{O}_{8+\delta}{ }_{39,40}$.

Compared to intralayer coupling, the interlayer coupling in vdW materials is relatively weak due to its vdW type. Many physical properties sensitive to the interlayer spacing have been engineered via the out-of-plane strain ${ }^{41}$. For example, out-of-plane strain can introduce positive piezoconductive effect in few-layer graphene, which is inaccessible through in-plane strain engineering ${ }^{42}$, and enhance the electrostatic potential from the substrate in graphene/hBN heterostructures, enabling the dynamical tuning of the band structure ${ }^{43}$. Moreover, the reduction of interlayer spacing induced by out-of-plane strain in magic-angle twisted bilayer graphene successfully varies the interlayer hopping energy, leading to the shift of the magic angle for the superconducting and correlated insulating states ${ }^{44}$. Other interlayer couplings that could be effectively modified by out-of-plane strain include interlayer exchange energy, which controls the interlayer magnetic order in few-layer $\mathrm{Cr}_{3}{ }^{45,46}$, and interlayer exciton binding energy that determines the excitonic/optoelectronic behaviors ${ }^{47}$. Currently, the setup for applying out-of-plane strain on the vdW materials and heterostructures has already been well established. The future focus of out-of-plane strain engineering on vdW materials is twofold: exploring new states that are absent in ambient pressure, and understanding the mechanisms of novel interlayer effects. Here, examples of emergent interlayer effects include proximity spin-orbit coupling in graphene/TMDs heterostructures ${ }^{48,49}$, in-plane orbital effect in twisted double

${ }^{1}$ National Laboratory of Solid State Microstructures, School of Physics, Collaborative Innovation Center of Advanced Microstructures, Nanjing University, Nanjing, China. ${ }^{2}$ Institute of Interdisciplinary Physical Sciences, School of Science, Nanjing University of Science and Technology, Nanjing, China. ${ }^{\circledR}$ email: miao@nju.edu.cn; bincheng@njust.edu.cn 


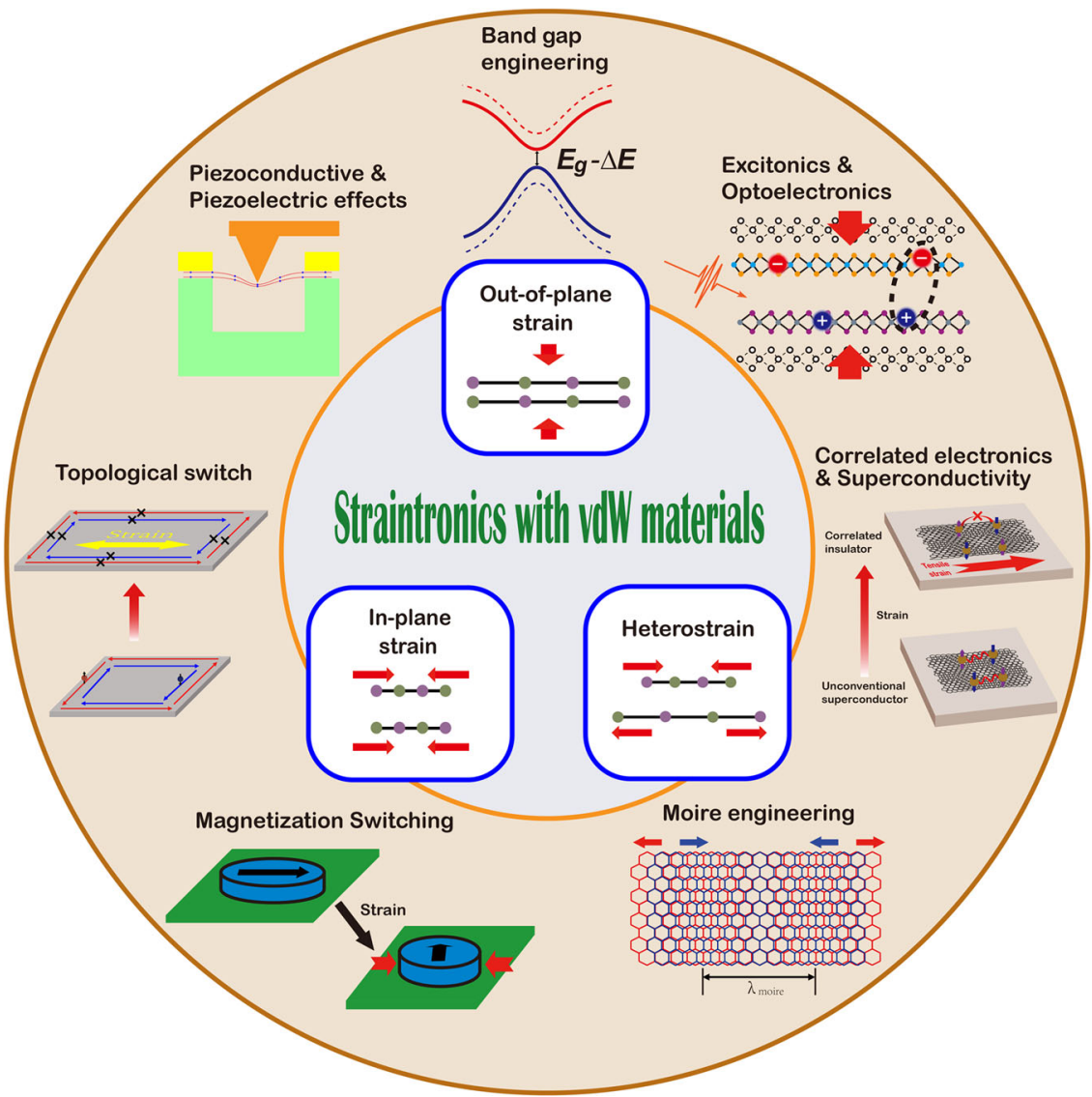

Fig. 1 Summary of straintronics with vdW materials. The strain engineering on vdW materials can be classified into in-plane strain, out-ofplane strain, and heterostrain. Related directions for the future research in this field are proposed.

bilayer graphene $e^{50}$, unconventional ferroelectricity in moiré heterostructures ${ }^{51,52}$, etc.

As a ubiquitous type of strain in vdW heterostructures, heterostrain arises when the adjacent 2D layers have unequal in-plane strains ${ }^{53-57}$. On one hand, heterostrain inherits certain characteristics of in-plane strain, such as the capability of tuning intralayer hopping/exchange energy and breaking rotational symmetry. On the other hand, heterostrain also possesses many features similar to interlayer strain, since heterostrain could modulate the interlayer coupling by altering the relative displacement of the adjacent layers. Notably, the relative interlayer displacement can adjust the lattice mismatch of the adjacent layers, enabling the design of diverse moiré patterns by engineering the heterostrain. This moiré engineering promises a versatile platform for the exploration of correlated electronics and photonics in not only 2D moiré systems, but also one-dimensional moiré superlattices in twisted bilayer graphene or TMDs ${ }^{58,59}$. Currently, heterostrain in vdW heterostructures is mainly generated by the inevitable thermal relaxation during the layer-stacking process, which leads to inhomogeneous and uncontrollable strain distribution. Such disorder of heterostrain distribution not only hinders the experimental investigation on the role of heterostrain engineering on correlated electronic behaviors in twisted bilayer graphene and TMDs, but also obstructs the extension of heterostrain engineering to other types of vdW heterostructures. To that end, developing new techniques to fabricate uniform and designable heterostrain in vdW heterostructures is one of the major challenges.

Another key challenge in this field is to develop fast and noninvasive methods to quantitatively characterize various types of strains in vdW materials, either naturally occurring or engineered.
Compared to transmission electron microscopes (TEM) or scanning tunneling microscopy (STM), optical techniques such as Raman spectroscopy could provide more efficient and mild strategies, and have been widely used in characterizing in-plane strain in monolayer graphene ${ }^{60,61}$ and TMDs ${ }^{62}$. Therefore, extending those optical measurement techniques to the characterization of in-plane strain in more vdW materials and heterostrain in vdW heterostructures is urgently required.

To sum up, strain engineering provides powerful means for tailoring quantum and topological properties in vdW materials by directly modulating the interatomic distances and rotational symmetry. Meanwhile, the emergence of thousands of new vdW materials and heterostructures provides tremendous opportunities for the research of straintronics. Those materials that could be immediately investigated include vdW semiconductors for band gap engineering and piezoconductive/piezoelectric effects, heterostructures consisting of vdW semiconductors for excitonics/ optoelectronics, twisted bilayer graphene, or TMDs for moire engineering and correlated electronics/superconductivity, vdW magnetic materials for magnetization switching, vdW topological materials for topological switching, etc. Aside from the motivations of fundamental researches, other targets could be to develop new types of strain-assisted device functionality, such as data sensing based on excitonics/optoelectronics, logic operation based on band engineering and topological switching, data storing and computing based on magnetization switching, and eventually achieve strain-assisted in-sensor memory and insensor computing devices desirable for applications such as etextiles, e-tattoo, etc., in flexible electronics. 
Received: 4 February 2021; Accepted: 19 May 2021; Published online: 17 June 2021

\section{REFERENCES}

1. Deng, S., Sumant, A. V. \& Berry, V. Strain engineering in two-dimensional nanomaterials beyond graphene. Nano Today 22, 14-35 (2018).

2. Dai, Z., Liu, L. \& Zhang, Z. Strain engineering of 2D materials: issues and opportunities at the interface. Adv. Mater. 31, 1805417 (2019).

3. Akinwande, D., Petrone, N. \& Hone, J. Two-dimensional flexible nanoelectronics. Nat. Commun. 5, 5678 (2014).

4. Bunch, J. S. et al. Electromechanical resonators from graphene sheets. Science 315, 490-493 (2007)

5. Lee, C., Wei, X., Kysar, J. W. \& Hone, J. Measurement of the elastic properties and intrinsic strength of monolayer graphene. Science 321, 385-388 (2008).

6. Bao, W. et al. Controlled ripple texturing of suspended graphene and ultrathin graphite membranes. Nat. Nanotechnol. 4, 562-566 (2009).

7. Guinea, F., Katsnelson, M. I. \& Geim, A. K. Energy gaps and a zero-field quantum Hall effect in graphene by strain engineering. Nat. Phys. 6, 30-33 (2010).

8. Levy, N. et al. Strain-induced pseudo-magnetic fields greater than 300 tesla in graphene nanobubbles. Science 329, 544-547 (2010).

9. Wang, Y. et al. Super-elastic graphene ripples for flexible strain sensors. ACS Nano 5, 3645-3650 (2011).

10. Zhao, J., Zhang, G.-Y. \& Shi, D.-X. Review of graphene-based strain sensors. Chin. Phys. B 22, 057701 (2013)

11. Miao, T., Yeom, S., Wang, P., Standley, B. \& Bockrath, M. Graphene Nanoelectromechanical systems as stochastic-frequency oscillators. Nano Lett. 14 2982-2987 (2014).

12. Teague, M. L. et al. Evidence for strain-induced local conductance modulations in single-layer graphene on $\mathrm{SiO}_{2}$. Nano Lett. 9, 2542-2546 (2009).

13. Huang, M., Yan, H., Heinz, T. F. \& Hone, J. Probing strain-induced electronic structure change in graphene by Raman spectroscopy. Nano Lett. 10, 4074-4079 (2010).

14. Zhao, J. et al. Ultra-sensitive strain sensors based on piezoresistive nanographene films. Appl. Phys. Lett. 101, 063112 (2012).

15. Zhang, $\mathrm{H}$. et al. Transport in suspended monolayer and bilayer graphene under strain: a new platform for material studies. Carbon 69, 336-341 (2014).

16. Li, S.-Y., Su, Y., Ren, Y.-N. \& He, L. Valley polarization and inversion in strained graphene via pseudo-Landau levels, valley splitting of real Landau levels, and confined states. Phys. Rev. Lett. 124, 106802 (2020).

17. $\mathrm{Ni}, \mathrm{Z}$. H. et al. Uniaxial strain on graphene: Raman spectroscopy study and bandgap opening. ACS Nano 2, 2301-2305 (2008)

18. Yang, R. et al. Tuning optical signatures of single- and few-layer $\mathrm{MoS}_{2}$ by blown-bubble bulge straining up to fracture. Nano Lett. 17, 4568-4575 (2017).

19. Zhang, Z. et al. Strain-modulated bandgap and piezo-resistive effect in black phosphorus field-effect transistors. Nano Lett. 17, 6097-6103 (2017)

20. Jiang, S., Xie, H., Shan, J. \& Mak, K. F. Exchange magnetostriction in twodimensional antiferromagnets. Nat. Mater. 19, 1295-1299 (2020).

21. Zhuang, H. L., Kent, P. R. C. \& Hennig, R. G. Strong anisotropy and magnetos triction in the two-dimensional Stoner ferromagnet $\mathrm{Fe}_{3} \mathrm{GeTe}_{2}$. Phys. Rev. B 93, 134407 (2016).

22. Šiškins, M. et al. Magnetic and electronic phase transitions probed by nanomechanical resonators. Nat. Commun. 11, 2698 (2020).

23. Wang, Y. et al. Strain-sensitive magnetization reversal of a van der Waals magnet. Adv. Mater. 32, 2004533 (2020)

24. Webster, L. \& Yan, J.-A. Strain-tunable magnetic anisotropy in monolayer $\mathrm{CrCl}_{3}$ $\mathrm{CrBr}_{3}$, and $\mathrm{Crl}_{3}$. Phys. Rev. B 98, 144411 (2018)

25. Wu, Z., Yu, J. \& Yuan, S. Strain-tunable magnetic and electronic properties of monolayer Crl3. Phys. Chem. Chem. Phys. 21, 7750-7755 (2019).

26. Son, J., Kim, K.-H., Ahn, Y. H., Lee, H.-W. \& Lee, J. Strain engineering of the berry curvature dipole and valley magnetization in monolayer $\mathrm{MoS}_{2}$. Phys. Rev. Lett. 123, 036806 (2019).

27. Zhang, S.-H. \& Liu, B.-G. Anisotropic rashba effect and charge and spin currents in monolayer BiTel by controlling symmetry. Phys. Rev. B 100, 165429 (2019).

28. Deng, Y. et al. Quantum anomalous Hall effect in intrinsic magnetic topological insulator $\mathrm{MnBi}_{2} \mathrm{Te}_{4}$. Science 367, 895-900 (2020).

29. Sharpe, A. L. et al. Emergent ferromagnetism near three-quarters filling in twisted bilayer graphene. Science 365, 605-608 (2019).

30. Serlin, M. et al. Intrinsic quantized anomalous Hall effect in a moiré heterostructure. Science 367, 900-903 (2020).

31. $\mathrm{Wu}, \mathrm{S}$. et al. Observation of the quantum spin Hall effect up to 100 kelvin in a monolayer crystal. Science 359, 76-79 (2018).
32. Liu, J., Qian, X. \& Fu, L. Crystal field effect induced topological crystalline insulators in monolayer IV-VI semiconductors. Nano Lett. 15, 2657-2661 (2015).

33. Malinowski, P. et al. Suppression of superconductivity by anisotropic strain near a nematic quantum critical point. Nat. Phys. 16, 1189-1193 (2020).

34. Cao, Y. et al. Unconventional superconductivity in magic-angle graphene superlattices. Nature 556, 43-50 (2018).

35. Liu, X. et al. Tunable spin-polarized correlated states in twisted double bilayer graphene. Nature 583, 221-225 (2020).

36. $\mathrm{Xi}, \mathrm{X}$. et al. Ising pairing in superconducting $\mathrm{NbSe}_{2}$ atomic layers. Nat. Phys. 12, 139-143 (2016).

37. Fatemi, V. et al. Electrically tunable low-density superconductivity in a monolayer topological insulator. Science 362, 926-929 (2018).

38. Chen, $\mathrm{C}$. et al. Atomic line defects and zero-energy end states in monolayer $\mathrm{Fe}(\mathrm{Te}$, Se) high-temperature superconductors. Nat. Phys. 16, 536-540 (2020).

39. $\mathrm{Yu}, \mathrm{Y}$. et al. High-temperature superconductivity in monolayer $\mathrm{Bi}_{2} \mathrm{Sr}_{2} \mathrm{CaCu}_{2} \mathrm{O}_{8+\delta}$. Nature 575, 156-163 (2019).

40. Zhao, S. Y. F. et al. Sign-reversing Hall effect in atomically thin high-temperature $\mathrm{Bi}_{2.1} \mathrm{Sr}_{1.9} \mathrm{CaCu}_{2.0} \mathrm{O}_{8+\delta}$ superconductors. Phys. Rev. Lett. 122, 247001 (2019).

41. Xiang, Z. J. et al. Pressure-induced electronic transition in black phosphorus. Phys. Rev. Lett. 115, 186403 (2015).

42. $\mathrm{Xu}, \mathrm{K}$. et al. The positive piezoconductive effect in graphene. Nat. Commun. 6 8119 (2015).

43. Yankowitz, M. et al. Dynamic band-structure tuning of graphene moiré superlattices with pressure. Nature 557, 404-408 (2018).

44. Yankowitz, M. et al. Tuning superconductivity in twisted bilayer graphene. Science 363, 1059-1064 (2019).

45. Song, T. et al. Switching 2D magnetic states via pressure tuning of layer stacking Nat. Mater. 18, 1298-1302 (2019).

46. $\mathrm{Li}, \mathrm{T}$. et al. Pressure-controlled interlayer magnetism in atomically thin $\mathrm{Crl}_{3}$. Nat Mater. 18, 1303-1308 (2019).

47. Xia, J. et al. Strong coupling and pressure engineering in $\mathrm{WSe}_{2}-\mathrm{MoSe}_{2}$ heterobilayers. Nat. Phys. 17, 92-98 (2021).

48. Island, J. O. et al. Spin-orbit driven band inversion in bilayer graphene by van der Waals proximity effect. Nature 571, 85-89 (2019)

49. Avsar, A. et al. Spin-orbit proximity effect in graphene. Nat. Commun. 5, 4875 (2014).

50. Lee, J. Y. et al. Theory of correlated insulating behaviour and spin-triplet superconductivity in twisted double bilayer graphene. Nat. Commun. 10, 5333 (2019).

51. Zheng, Z. et al. Unconventional ferroelectricity in moiré heterostructures. Nature 588, 71-76 (2020)

52. Yasuda, K., Wang, X., Watanabe, K., Taniguchi, T. \& Jarillo-Herrero, P. Stackingengineered ferroelectricity in bilayer boron nitride. Science https://doi.org/ 10.1126/science.abd3230 (2021).

53. Qiao, J.-B., Yin, L.-J. \& He, L. Twisted graphene bilayer around the first magic angle engineered by heterostrain. Phys. Rev. B 98, 235402 (2018).

54. Huder, L. et al. Electronic spectrum of twisted graphene layers under heterostrain Phys. Rev. Lett. 120, 156405 (2018).

55. Zhang, $C$. et al. Strain distributions and their influence on electronic structures of $\mathrm{WSe}_{2}-\mathrm{MoS}_{2}$ laterally strained heterojunctions. Nat. Nanotechnol. 13, 152-158 (2018).

56. Jiang, Y. et al. Charge order and broken rotational symmetry in magic-angle twisted bilayer graphene. Nature 573, 91-95 (2019).

57. Shi, $\mathrm{H}$. et al. Large-area, periodic, and tunable intrinsic pseudo-magnetic fields in low-angle twisted bilayer graphene. Nat. Commun. 11, 371 (2020).

58. Bai, Y. et al. Excitons in strain-induced one-dimensional moiré potentials at transition metal dichalcogenide heterojunctions. Nat. Mater. 19, 1068-1073 (2020)

59. Timmel, A. \& Mele, E. J. Dirac-Harper theory for one-dimensional moiré superlattices. Phys. Rev. Lett. 125, 166803 (2020)

60. Lee, J. E., Ahn, G., Shim, J., Lee, Y. S. \& Ryu, S. Optical separation of mechanical strain from charge doping in graphene. Nat. Commun. 3, 1024 (2012).

61. Mueller, N. S. et al. Evaluating arbitrary strain configurations and doping in graphene with Raman spectroscopy. 2D Mater. 5, 015016 (2017)

62. Michail, A., Delikoukos, N., Parthenios, J., Galiotis, C. \& Papagelis, K. Optica detection of strain and doping inhomogeneities in single layer $\mathrm{MoS}_{2}$. Appl. Phys. Lett. 108, 173102 (2016).

\section{ACKNOWLEDGEMENTS}

This work was supported by National Natural Science Foundation of China (12074176 61625402, 62034004, 61921005, 61974176), and the Collaborative Innovation Center of Advanced Microstructures and Natural Science Foundation of Jiangsu Province (BK20190276, BK20180330), Fundamental Research Funds for the Central Universities (020414380084). 


\section{AUTHOR CONTRIBUTIONS}

F.M., B.C., and S.J.L co-wrote the manuscript.

\section{COMPETING INTERESTS}

The authors declare no competing interests.

\section{ADDITIONAL INFORMATION}

Correspondence and requests for materials should be addressed to F.M. or B.C.

Reprints and permission information is available at http://www.nature.com/ reprints

Publisher's note Springer Nature remains neutral with regard to jurisdictional claims in published maps and institutional affiliations. (i) Open Access This article is licensed under a Creative Commons (c) Attribution 4.0 International License, which permits use, sharing, adaptation, distribution and reproduction in any medium or format, as long as you give appropriate credit to the original author(s) and the source, provide a link to the Creative Commons license, and indicate if changes were made. The images or other third party material in this article are included in the article's Creative Commons license, unless indicated otherwise in a credit line to the material. If material is not included in the article's Creative Commons license and your intended use is not permitted by statutory regulation or exceeds the permitted use, you will need to obtain permission directly from the copyright holder. To view a copy of this license, visit http://creativecommons. org/licenses/by/4.0/.

(c) The Author(s) 2021 